were told that she had had four more convulsions. The skin being still very dry, and very little urine having been passed, and that into the bed, we gave her a hypodermic injection of \& quarter of a grain of pilocarpine at 1.45 P.M. At 7 P. M. she was still unconscious, but was perspiring pro. fusely, and had also retained some milk and soda-water, all previous attempts at feeding having been unsuccessful. On the morning of the $16 \mathrm{th}$, forby-eight hours after the com. mencement of the coma, she became conscious, and then complained of severe headache and complete amaurosis. She was then pat on twenty-grain doses of citrate of potash every four hours. In the course of the day she passed urine, which on examination contained one sixth of albumen, which has since almost disappeared. She then made an aninterrupted recovery, regaining her sight in twenty-four hours, and at the present is up and attending to her household duties, and is suckling the infant, which is also quite well.

The interest attaching to the case lies in the success which attended the treatment, as regards both the child and the mother. Knowing, as everyone does, the almost aninterrupted fatality of cases occurring before labour, it is with the greatest pleasure that we put on record a case in which not only the mother completely recovered, but the child also survived. We wish to add our testimony to the advisability of immediate operative treatment in all cases occurring late in pregaancy, the nature of the procedure of course depending on the severity of the case. Inasmuch as in our case the convulsions themselves were not very frequent, we decided to bring on labour gradually; but, in a case in which the convulsions threatened to terminate life by their frequency and severity, we should not hesitate to ampty the uterus by dilatation with Hegar's dilators.

Cambridge-gardens, $\mathbf{W}$.

\section{A CASE OF ENTERIC FEVER,}

IN WHICH SYMPTOMS OF PYAMIA APPEARED ON THE THIRTIETH AND DEATH OCCURRED ON THE

THIRTY.SEYENTH DAY OF ILLNESS.

By IAIN Clerk, M.B.,

PHYSICIAN-SUPERINTENDENT TO THE JOINT BURGHS FEVER HOSPITAL, KNIGITSWOOD, GLASGOW.

THz formation of small abscesses in certain parts of the body is not unusual in enteric fever, especially if the case has been a long-drawn-out one, or when rapid emaciation has taken place. With regard to abscesses, Murchison has the following note: "I have met with many instances in which, during convalescence, abscesses have formed beneath the skin in different parts of the body. ...... In rarer instances pus is deposited in the jolnts or in some of the internal organs, and then the case usually terminates fatally." The following short account of a case in which symptoms of pyrmia appeared on the thirtieth and death occurred on the thirty-seventh day of illness is, I think, worthy of record, seeing such a complication is somewhat anusual in this disease.

J. K-, aged twenty-eight, labourer, was admitted to the Joint Burghs Hospital on Sept. 30ih, 1891, cerbified as suffering from enteric fever. His illness began eight days before admiscion, with headache, shivering, vomiting, general weakness, \&e. It is not necessary to follow the history of the case, as there was nothing special to note, with the exception, perhaps, that the crop of rose papules was very profuse over the abdomen and chest. The highest temperature recorded was $103 \cdot 8^{\circ} \mathrm{F}$. There was no looseness of the bowels, although from colour and odour the stools were characteristic enough. The temperature, which all through his illness was very steady, became variable after the twenty-sixth, ${ }^{1}$ dropping on the twenty-ninth to $98^{\circ}$ in the morning, but rising in the evening to $103^{\circ}$. Thirtieth day : Morning temperabure $103^{\circ}$, evening $1018^{\circ}$. Thirtyfirst: Morning temperature $102^{\circ}$, evening $104^{\circ}$; and after this it ranged between $102^{\circ}$ and $104^{\circ}$, or sometimes slightly over.

On the thirtieth day of illness the right cheek was seen to bulge a little, and on the following day the right eye was completely closed, the upper and lower eyelids being largely swollen. Oz the thirty. third day the left eye became involved, botil lids swollen enormously, and the eye closed.

I mean day of illnces, not day of the month, so much in vogue, but aractically valueless.
On the thirty-fifth day a large swelling, not glandular, appeared in both sides of the neck at the angles of the jaws, and by this time the outer half of the right upper eyelid sloughed away, and there was considerable bogginess in the right temporal fossa and over the right ear. In all of those places named collections of pus took place with alarming rapidity ; and although openings and counter-openings were made freely and early, sloughings of portions of both upper eyelids took place, and the patient gradually sank, and died on the thirty-seventh day of illness. As the temperature did not drop at the end of three weeks, it is likely that inflammatory action was still going on in the lower part of the small bowel; and it is conceivable that some purulent matter from an ulcerating patch may have got into the circulation, and, pos sibly carrying the enteric poison with it, given rise to those abscesses of which I hare spoken. From the absence of glandular enlargement, it seems to have passed by the blood circulatory rather than the lymphatic system. Glasgow.

\section{A CASE OF HYOSCINE POISONING TREATED BY PILOCARPINE.}

By Herbert L. Evans, M.B., C.M. Edin.

IN connexion with the case of poisoning by byoscine and that of poisoning by pilocarpine mentioned on pages 328 and 329 respectively of THE LANCET of Feb. 6th, 1892, the following may be of interest:-

On Oct. 16th, 1890, I was called to J. B-, a case of delirium tremens, at 4.45 A.M. He had just had a fit. This, in his case, was no unusual occurrence, his first attack having begun on Sept. $20 \mathrm{ch}$ of the same year with an epileptiform seizure. Chloral, bromides, and morphia had previously failed to produce sleep, so a two-minim injection of a 1 per cent. solution of hyoscine was given, but this also failed. Eight hours later he had nearly three minims by the mouth, with no better result. In the evening morphia and chloral were given without effect. Later on, as he could not be kept in bed and was quite unmanageable, a three-minim injection of hyoscine of presumably the same strength, but from a different bottle, was given. The patient rapidly became comatose with dilated pupils and arteries, rapid pulse, congested face, hot, dry skie, and rapid and deep breabhing. Morphia was injected, but without any apparent improvement, Directing that the patient should be energetically stimulated by pinching, I hurried to the surgery, and on returning with pilocarpine found that, as before, the patient could not be roused. Unfortunately no record of the injections of this was kept. So urgent zeemed the necessity of producing symptoms approsching those characteristic of the drug that injections were made in the thighs and arms at intervals of about five minutes. In a short time there was a faint reaction of the pupils to light, with a tendency to moistness of skin, whilst the breathing and pulse improved; and on my next holding the candje to the face I was gratified by seeing the patient knock it from my hand, after which rapid improvement took place, followed by complete recovery. Great soreness and itching were complained of next day, the latter relieved by alkaline washes and cocaine ointment. His thighs and arms showed marks of about eight punctures, and he had probably had about two grains of nitrate of pilocarpine. His general health improved under strychnine, red extract of cinchona, \&c., su that in a short time he was able to indulge in shooting and billiards, and seemed to have no craving for liquor.

Goring-on-Thames.

Hospital For Srck CHILdREN AND WOMEN, BRISTOL. - The year 1891 completed the twenty-fifth year of thisinstitution, and theannual report just issued records some interesting details on the history and career of the charity covering that period. In 1866 the hospital opened with six cots. The beds now number 100 . The medical statistics of the past year show that more patients were treated both in the hospital and as out-door patients than in any former twelve months. The average annual expenditure is estimated now at $£ 3300$. The year's income from all sources (except legacies) fell short of the expenditure by $£ 1516 s .10 d$ The deficio had, bowever, been discharged out of a legacy received of $£ 296414 s$. $7 d$; the current year consequently began freed of an adverse balance. 